\title{
Development of Fully-Distributed Fiber Sensors Based on Brillouin Scattering
}

\author{
Xuping ZHANG, Yuangang LU, Feng WANG, Hao LIANG, and Yixin ZHANG \\ Institute of Optical Communication Engineering, Nanjing University, Nanjing 210093, China \\ *Corresponding author: Xuping Zhang E-mail: xpzhang@nju.edu.cn
}

\begin{abstract}
Brillouin scattering based optical fiber sensors (BOFS) have the unique advantages over other sensors such as long distance, fully distributed, and multi-parameter sensing. The progresses on the development of BOFS technology in Nanjing University are reviewed. The key technologies to make BOFS with ultra-long distance, high spatial resolution, and fast measuring speed are discussed and realized.
\end{abstract}

Keywords: Brillouin scattering, fully distributed optical fiber sensor, Brillouin optical time domain reflectometry, strain monitoring

\section{Introduction}

The Brillouin scattering based optical fiber sensor (BOFS) can realize fully distributed strain and temperature measurement along the fiber and by one end of the fiber, which provides a convenient way for health monitoring on the large civil structures, such as dams, bridges, pipe lines, and tunnels, for which long sensing length is required to cover the entire structures in $2 \mathrm{D}$ or $3 \mathrm{D}$. Therefore, BOFS has gained extensive attention and many BOFS systems based on optical time-domain reflectometry (BOTDR) are developed in recent years [1-11]. However the existed BOFS systems can't meet the requirement of some specific situations. For example, the spatial resolution of measurements is limited to $1 \mathrm{~m}$ which is difficulty to detect crack in concrete structures, while the sensing time is usually longer than minutes which can't be used as real-time monitoring for invasion. We have focused on the improvement of performances for BOFS system including spatial resolution, measurement time, and sensing range since 2008 [12-17]. This paper reports some novel proposals we have made.

\section{Improvement of spatial resolution}

Since the spatial resolution of BOFS is limited to $1 \mathrm{~m}$ by the phonon life time of $10 \mathrm{~ns}[18]$, the application of BOFS in structural health was limited. We proposed a novel multi-Lorentz fitting method based on equivalent optical pulse (EOP) to improve the spatial resolution. In this method, the EOP is defined as a nominal optical pulse which is obtained by integrating the probe optical pulse over the time to accomplish one single sampling. So the Brillouin signal obtained in one single sampling can be treated as if a Brillouin signal generated by the EOP and received by the system within infinite short time. The received Brillouin backscattered spectrum (BBS) of EOP is decomposed into sub-BBS's according to the shape of EOP. Then the BBS is fitted by multi-Lorentz function to obtain central frequency of each sub-BBS. The strain/temperature in the

Received: 9 October 2010 / Revised version: 8 November 2010

(C) The Authors(s) 2010.This article is published with open access at Springerlink.com 
length corresponding to each sub-BBS can be obtained by use of the dependence of Brillouin frequency shift on strain /temperature.

The BBS received by the receiver contains the total information generated by probe pulse light (PPL) in the fiber corresponding to a half length of PPL. But it takes time to receive BBS due to the restriction of analog-to-digital (A/D) converter's sampling rate. Thus the received BBS is an integration of BBS over the half length of PPL and the half length which the PPL transmits in the receiving time. So the EOP should be considered as the integration of probe pulse when it transmits along the fiber in the receiving time.

The probe pulse is assumed as an ideal rectangular pulse described as

$$
y_{0}(t)= \begin{cases}A & \left(t_{0} \leq t \leq t_{0}+\tau\right) \\ 0 & \left(t<t_{0} \text { or } t>t_{0}+\tau\right)\end{cases}
$$

where $\tau$ is the pulse width. If the receiving time needed for $\mathrm{A} / \mathrm{D}$ converter to complete one single sampling is $\tau^{\prime}$, according to the difference between the pulse width and the receiving time, the corresponding EOP's are described as (2) and (3), respectively and shown in Fig. 1 .

(a) if $\tau \geq \tau^{\prime}:$
$y(t)= \begin{cases}A\left(t-t_{0}\right) & \left(t_{0} \leq t<t_{0}+\tau^{\prime}\right) \\ A \tau^{\prime} & \left(t_{0}+\tau^{\prime} \leq t \leq t_{0}+\tau\right) \\ A \tau^{\prime}-A\left(t-t_{0}-\tau\right) & \left(t_{0}+\tau<t \leq t_{0}+\tau+\tau^{\prime}\right) \\ 0 & \text { (others) }\end{cases}$

(b) if $\tau \leq \tau^{\prime}$ :

$$
y(t)= \begin{cases}A\left(t-t_{0}\right) & \left(t_{0} \leq t<t_{0}+\tau\right) \\ A \tau & \left(t_{0}+\tau \leq t \leq t_{0}+\tau^{\prime}\right) \\ A \tau-A\left(t-t_{0}-\tau^{\prime}\right) & \left(t_{0}+\tau^{\prime}<t \leq t_{0}+\tau+\tau^{\prime}\right) \\ 0 & (\text { others })\end{cases}
$$

It is shown that the width of the EOP is $\tau+\tau^{\prime}$, and the corresponding spatial resolution is

$$
\Delta z^{\prime}=\frac{c\left(\tau+\tau^{\prime}\right)}{2 n} .
$$

The BBS can be described as a Lorentzian curve, which is given as

$$
g\left(v, v_{B}\right)=\frac{p_{0}(\omega / 2)^{2}}{\left(v-v_{B}\right)^{2}+(\omega / 2)^{2}}
$$

where $v$ is the frequency of $\mathrm{BBS}, v_{B}$ is the central frequency, $p_{0}$ is the peak power, and $\omega$ is the full width at half maximum (FWHM).

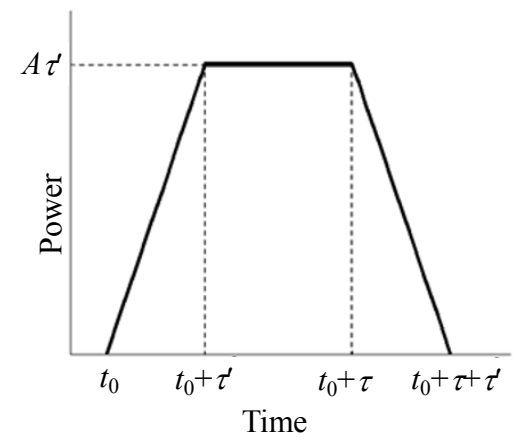

(a)

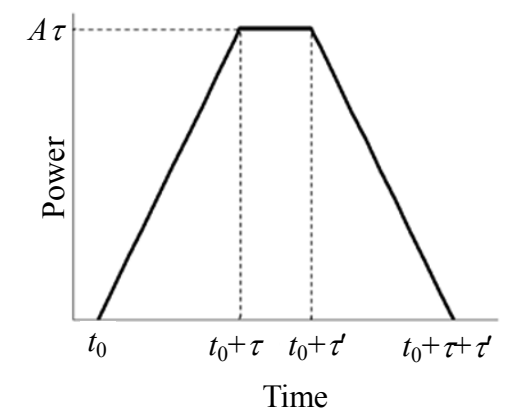

(b)

Fig. 1 (a) Equivalent optical pulse when $\tau \geq \tau^{\prime}$ and (b) equivalent optical pulse when $\tau \leq \tau^{\prime}$.

As the BBS contains the total information generated by PPL in the fiber corresponding to a half length of PPL, the PPL can be equally divided into $m$ segments and the BBS can be seen as the sum of $\mathrm{BBS}_{i}$ generated by $\mathrm{PPL}_{i}$ in different sections of fiber within the spatial resolution, where $i$ means different subscripts of BBS and PPL from 1 to $m$. If strain distribution within the spatial resolution is non-uniform, the BBS will be distorted. The distorted BBS can be described as the following equation:

$$
g\left(v, v_{B}\right)=\sum_{i=1}^{m} g_{i}\left(v, v_{B i}\right)=\sum_{i=1}^{m} \frac{p_{i}\left(\omega_{i} / 2\right)^{2}}{\left(v-v_{B_{i}}\right)^{2}+\left(\omega_{i} / 2\right)^{2}}(6)
$$

where $g_{i}\left(v, v_{B i}\right)$ is the BBSi, $v_{B i}$ is the central frequency of $\mathrm{BBS} i, p_{i}$ is the peak power of BBS $i$, and $\omega_{i}$ is the FWHM of BBSi. 
When $v_{B i}$ and $\omega_{i}$ are the same with $v_{B}$ and $\omega$, and the strain/temperature is constant, it is easily to obtain $p_{i}$ according the ratio of $p_{i}$ and $p$, as long as the shape of the equivalent optical pulse is known.

If the strain at one section of fiber is different from others and the length of the section is shorter than the width of EOP, $\quad p$ and $\omega$ change little with the strain. Therefore, it is feasible to consider they are constant, so we could obtain each $v_{B i}$ by iterative fitting from the section where the strain is known. At last, the strain curve along the fiber is obtained and the spatial resolution increases to $\frac{c\left(\tau+\tau^{\prime}\right)}{2 m n}$. In order to perform the iterative fitting process, the length of the divided fiber unit must be integer multiple of the sampling length.

The experimental result demonstrates that the spatial resolution for strain measurement is improved to $0.05 \mathrm{~m}$ by multi-Lorentz fitting method based on EOP [12].

\section{Increase of measuring speed}

Conventional BOFS system based on spontaneous BBS narrow-band detection is not only time-consuming but also controlling complicated in frequency-scanning. In addition, the weak spontaneous Brillouin scattered signals need to be averaged over thousands times to achieve the needed signal-to-noise ratio. Thus the time to complete a measurement generally requires tens minutes. We proposed several new methods to increase the measuring speed and make real-time monitoring in the past years $[16,17]$.

\subsection{Wideband detection of Brillouin scattering spectrum}

The schematic diagram of BBS wideband detection system is shown in Fig. 2.

A double-balanced photodetector with a $1 \mathrm{GHz}$ bandwidth is used as the wideband receiver to transform the whole beat optical signals into an electrical signal. A high-speed A/D converter with a
$1 \mathrm{GHz}$ bandwidth is used to convert analogue signals received from the photodetector to digital signals. The obtained signals are divided into sections according to the duration of probe pulse, and then each of them is transformed by discrete Fourier transform (DFT). As each of the signal-section is corresponding to a section of length along the sensing fiber, the power spectrum obtained by DFT just stands for the BSS that is generated when the probe pulse passes through the corresponding fiber section. By gathering all the BSS units along the sensing fiber, we can obtain a three-dimensional BSS, which includes distance, frequency, and power information. By fitting a Lorentzian function to each BSS, the Brillouin frequency shift and power change are deduced from the fitted curve. Temperature/strain change along the sensing fiber is determined by analyzing the frequency shift and power change of the total BSS.

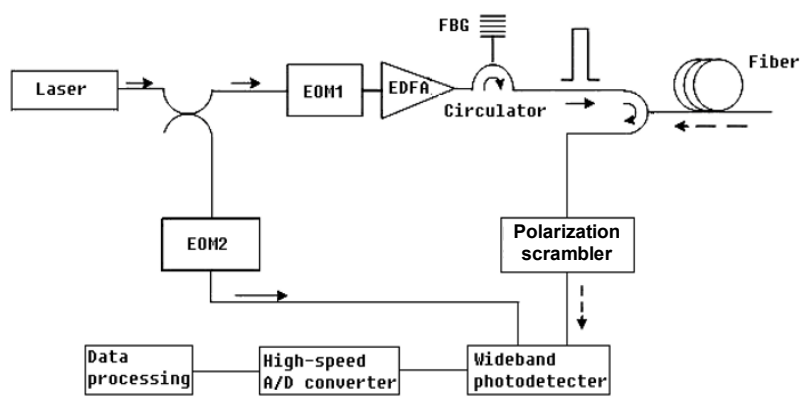

Fig. 2 Schematic configuration of BOTDR system used for wideband detection of BBS

The spatial resolution of the proposed BOFS system is determined by

$$
\delta z=\max \left\{\frac{c W}{2 n}, \frac{c N}{2 n f_{S}}\right\}
$$

where $c$ is the speed of light in vacuum, $n$ is the refractive index of fiber core, $w$ is the pulse width, $N$ is the number of DFT points, and $f_{S}$ is the sampling rate of high-speed $\mathrm{A} / \mathrm{D}$ converter.

As the proposed BOFS system doesn't need the process of scanning, the total measurement time is at least one order of magnitude smaller than that of the narrow-band detection BOFS system, though the 
data processing time of the proposed system may be a little longer than that of conventional system.

In order to test the effective of the proposed BSS wideband detection method, a distributed temperature measurement experiment is conducted. The total sensing length is made up of three sections of fiber fusion spliced together and arranged as shown in Fig. 3.

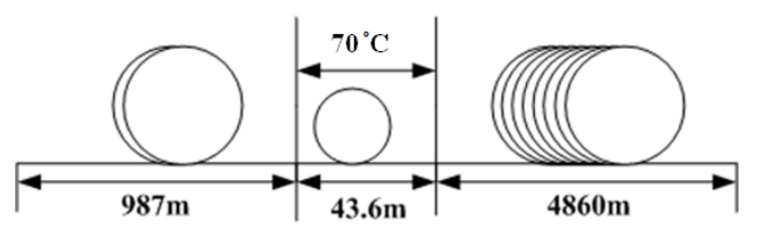

Fig. 3 Configuration of the sensing fiber in the experiment.

Figure 4 shows Brillouin frequency shift of the heated section of the fiber. Because in the experiment the pulse width was $20 \mathrm{~ns}$ and the sampling rate was $4 \mathrm{G} \mathrm{Sa} / \mathrm{s}$, the number of sampling points was 80 . The refractive index of the core of standard single mode fiber was 1.46 . Therefore, the spatial resolution of BOTDR system in the experiment was about $2 \mathrm{~m}$ according to (7). In Fig. 5 it could be seen that the length of the heated fiber was $44 \mathrm{~m}$, which was in good agreement with the actual value of $43.6 \mathrm{~m}$. According to the relationship between temperature and Brillouin frequency shift, the temperature of the heated fiber was $69.8{ }^{\circ} \mathrm{C}$, which was in good agreement with actual value of $70{ }^{\circ} \mathrm{C}$.

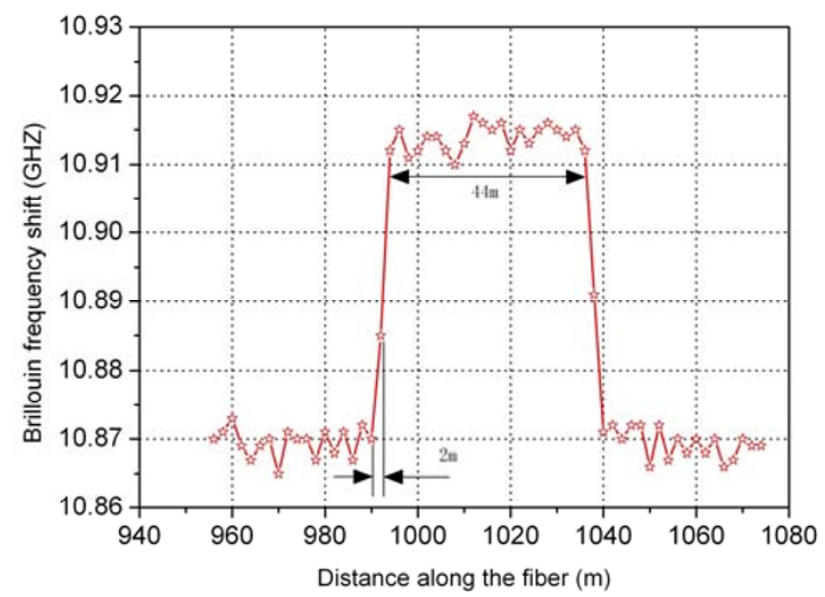

Fig. 4 Brillouin frequency shift of the heated section of the fiber.
BOTDR system based on SBSS wideband detection method has been demonstrated for a $6 \mathrm{~km}$ fiber, and a temperature resolution of $3{ }^{\circ} \mathrm{C}$ and a spatial resolution of $2 \mathrm{~m}$ have been achieved. The measurement time is only about one-tenth that of conventional narrow-band detection method.

\subsection{Real-time spectrum analysis}

Figure 5 shows the configuration of real-time spectrum analysis module.

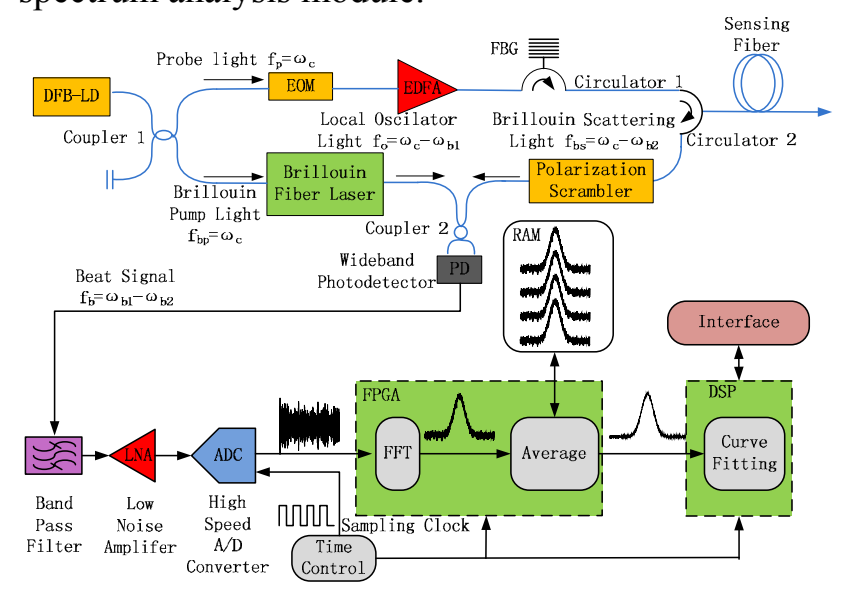

Fig. 5 Configuration of BOFS system based on real-time spectrum analysis.

Unlike the conventional BOFS system, the new method captures the whole frequency spectrum at one time rather than sweeping and measuring the spectrum from point to point. A narrow band distributed feedback laser diode (DFB-LD) with the center frequency of $\omega_{c}$ is used as light source. The light source is divided into two parts by coupler 1 . The first part is the probe light which is encoded by electrical optical modulator (EOM) and amplified by Erbium-doped fiber amplifier (EDFA). A fiber Bragg grating (FBG) and a circulator are used to filter the wide band noise of EDFA. The encoded light is then injected into the sensing fiber through circulator 2. The second part of light source acts as a pump light to stimulate a Brillourin fiber laser. The fiber laser generates a local oscillator with center frequency of $f_{10}=\omega_{c}-\omega_{b 1}$. The back scattering signal from circulator 2 passes through a polarization scrambler to get uniform distributed polarization 
state. The center frequency of the back scattering signal is $f_{b s}=\omega_{c}-\omega_{b 2}$. With coherent detection, the photo detector produces an electronic beat signal with center frequency of $f_{b}=\omega_{b 1}-\omega_{b 2}$.

After passing through the band pass filter and low noise amplifier, the beat signal is sampled by a $2.5 \mathrm{GHz}$ high speed A/D converter. The obtained time domain data is divided into groups and transformed to frequency domain with fast Fourier transform (FFT). Since single measurement's signal to noise ratio (SNR) is relatively low, average is needed to improve the SNR. All the FFT and average operations are done within a field programmable gate array (FPGA) device. As each group is corresponding to a section of length along the sensing fiber, the power spectrum obtained by FFT stands for the BBS that is generated during the probe pulse passes through the corresponding fiber section. Brillouin frequency shift and power change are deduced from curve fitting with Lorentzian function. The temperature/strain change along the sensing fiber is determined by analyzing the frequency shift and power change. A high speed digital signal processor (DSP) is used to do the curve fitting and data analyzing while maintaining the human-computer interface.

At least three advantages can be achieved with real-time spectrum analysis. First of all, since the proposed method doesn't need the process of frequency-scanning, the total measurement time is at least one order of magnitude smaller than that of traditional detection system. Although the total data processing amount of the proposed system is much larger than the conventional one, it can be solved with pipeline and parallel processing techniques. The second advantage is that the system's ability for capturing transient events is improved which can monitor rapidly changed signal. The third one is that the reliability of the system is enhanced since the complication of the detection system is reduced by abandoning the frequency-scanning components.

Contrast experiments are performed to verify our detection method's performance. The total sensing fiber is set up with three sections of fusion spliced fibers as shown in Fig. 6. The second section's temperature is controlled with a heater during the measurement. The heater is oscillating with a period of $90 \mathrm{~s}$. The pulse width is $10 \mathrm{~ns}$ and group size for FFT is 256 points. Thus the spatial resolution is approximate $10 \mathrm{~m}$. Figure 7 shows the Brillouin frequency shift of the heated section of the fiber obtained with conventional method.

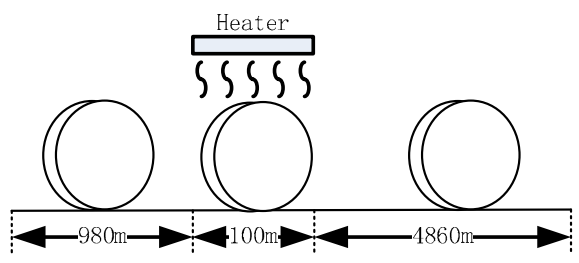

Fig. 6 Experimental setup.

The trace in Fig. 7 shows a $40 \mathrm{MHz}$ frequency shift of the heated section. However we can't see the oscillating process of the temperature since frequency-scanning is used which will cause dead zone during the measurement.

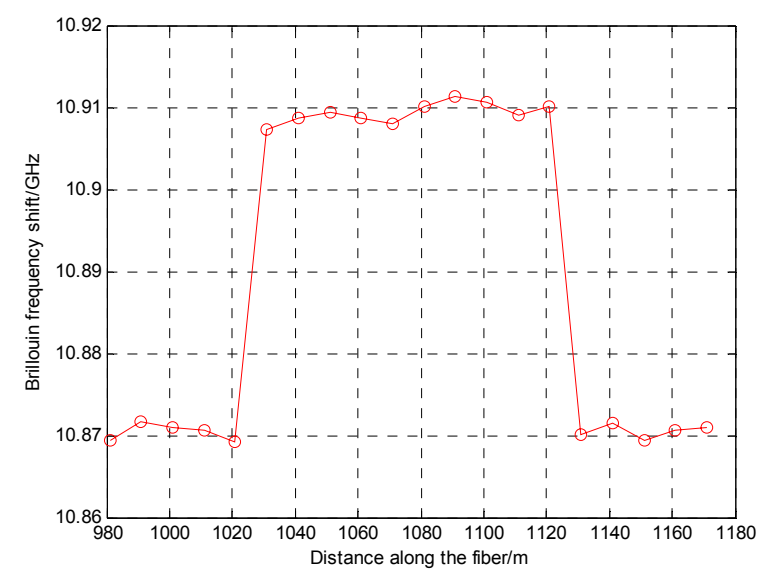

Fig. 7 Experimental result of conventional method.

Figure 8 shows the results obtained with the real-time spectrum analysis. During the same time, the proposed method completed 9 times measurements, which clearly displayed the temperature oscillating. The measured oscillating period is about $91.2 \mathrm{~s}$, which is in good agreement with the actual value. In practice, the fluctuation of temperature for the sensing fiber is a hint of the outside environment's fierce change which can act 
as an early warning for structure healthy monitoring.

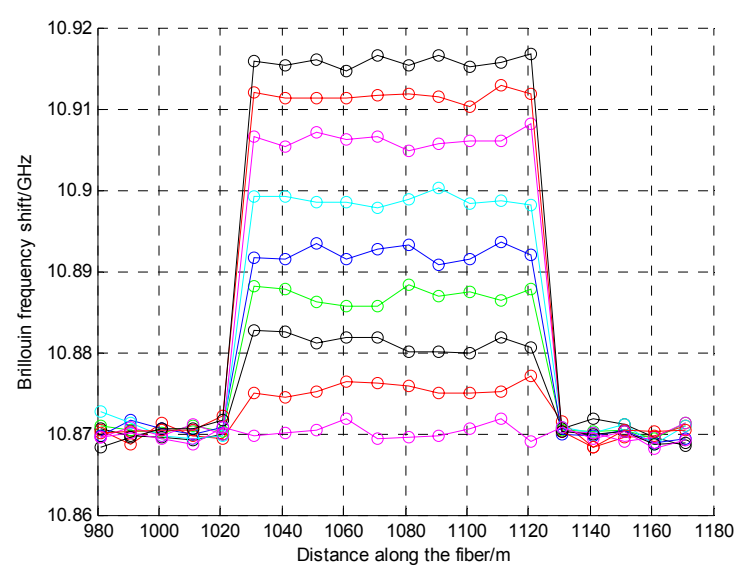

Fig. 8 Experimental result of proposed method.

\section{Enhancement of sensing range}

The sensing range of BOFS is highly determined by the SNR of the obtained Brillouin signal. Thus a long pulse which can provide higher Brillouin back scattering power must be used in the sensing system. However, according to (7), long pulse will decrease the spatial resolution of BOFS. Because of the tradeoff between sensing range and spatial resolution, we proposed to use coded probe pulses to improve the SNR of the measured signal. In our experiment, Hadamard coded pulse sequences were used. They can be easily generated from a bi-polar Hadamard matrix. And the sum of the auto-correlation of Hadamard matrix rows can be expressed as delta function. Thus, when using pulses formatted into the rows of Hadamard matrix, we can restore the signals by doing the cross-correlation between the backscattered signals and their corresponding rows. It is found that the recovered signal has a higher SNR compared with the one obtained by single pulse with the same peak power. The spatial resolution of coded BOFS is determined by the pulse width of the coded sequence unit. With this method we can achieve a high spatial resolution over a long sensing range [17].

A BOFS system which employs Hadamard sequence as the probe signal to increase the SNR of the system is proposed as shown in Fig. 9. Instead of injecting a single pulse into the sensing fiber to obtain the BBS, the BBS of the fiber is measured by injecting Hadamard sequence probe pulses. When being compared with the conventional BOFS system, the same sensing range can be achieved for the proposed system by using lower peak power pulse light, without reducing the spatial resolution.

In the experiment, $7 \mathrm{~km}$ and $20 \mathrm{~km}$ SMF28 fiber are connected by a $4 \mathrm{~km}$ dispersion shift fiber (DSF) to constitute the sensing fiber with a length of $31 \mathrm{~km}$, as shown in Fig. 10. Brillouin frequency shift of SMF28 and DSF are $10.87 \mathrm{GHz}$ and $10.72 \mathrm{GHz}$, respectively, at room temperature and in loose condition. 64-bit coded pulses with unit pulse width of $500 \mathrm{~ns}$ and peak power of $-4 \mathrm{dBm}$ are used as probe pulses.

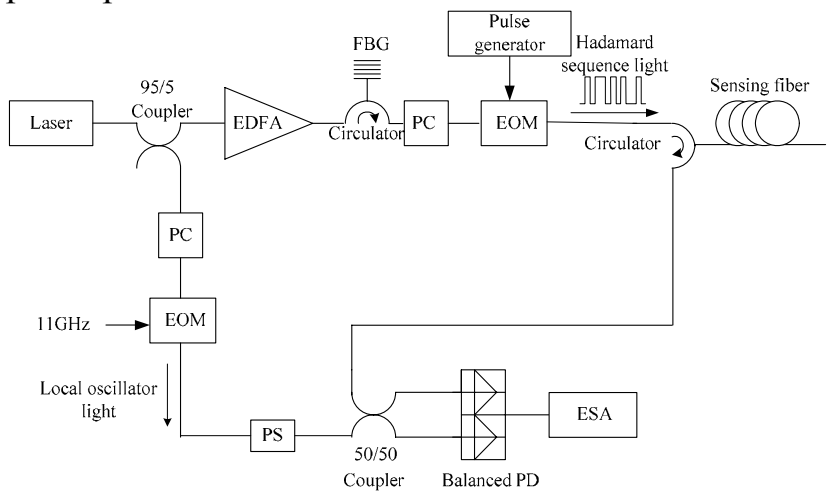

Fig. 9 Schematic diagram of the experimental setup.

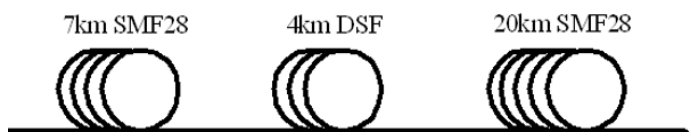

Fig. 10 Configuration of the sensing fiber in the experiment.

Figure 11 shows the restored time trace of Brillouin scattering signal corresponding to the frequency of $10.87 \mathrm{GHz}$ with frequency scanning method. It can be seen that three fiber sections are distinguished clearly. And the spatial resolution is $50 \mathrm{~m}$ corresponding to the unit pulse width $500 \mathrm{~ns}$. The SNR at the fiber end is approximately $15 \mathrm{~dB}$. According to the loss of the detected Brillouin signal, which is $0.4 \mathrm{~dB} / \mathrm{km}$, when the total sensing fiber length increases from $31 \mathrm{~km}$ to $61 \mathrm{~km}$, we can still get a SNR as high as $3 \mathrm{~dB}$. It indicates that the 
longest sensing range by use of the proposed method can be larger than $60 \mathrm{~km}$.

Experimental results verify the validity of our proposed coded pulses method. The dynamic range of BOTDR system can be increased without reducing the spatial resolution by using coded pulses as the probe pulse. Further, by this means the required peak power of the probe pulse can be much lower than that of the single pulse method. Hence it can avoid the unexpected nonlinear effects in the sensing fiber caused by probe pulse with high peak power as well.

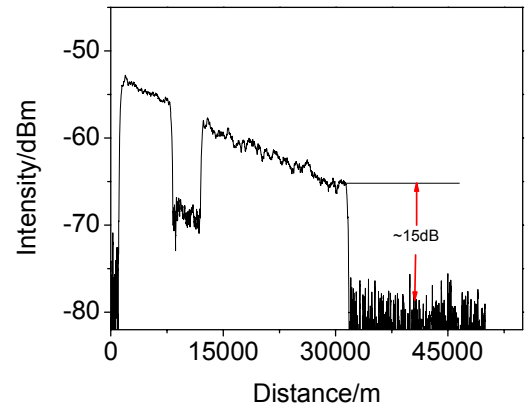

Fig. 11 Restored time trace of Brillouin scattering signal at $10.87 \mathrm{GHz}$.

\section{Conclusions}

Our work in improving the performances of BOFS system is reviewed. The improvement of spatial resolution by multi-Lorentz fitting method based on EOP, quick measurement method based on wideband detection of Brillouin scattering spectrum and real-time spectrum analysis, the enhancement of sensing range based on coded probe pulses are reported.

\section{Acknowledgment}

This work was supported by National Basic Research Program of China (973 Program) under Grant No. 2010CB327803 and National Natural Science Foundation of China (61027617).

\section{References}

[1] B. Lee, "Review of the present status of optical fiber sensors." Optical Fiber Technology, vol. 9, no. 2, pp. 57-79, 2003.
[2] A. Kobyakov, S. Kumar, D. Q. Chowdhury, et al. , "Design concept for optical fibers with enhanced SBS threshold," Optical Express, vol. 13, no. 14, pp. 5338-5346, 2005.

[3] G. P. Lees, P. C. Wait, M. J. Cole, and T. P. Newson, "Advances in Optical Fiber Distributed Temperature Sensing Using the Landau-Placzek Ratio", IEEE Photonics Technology Letters, vol. 10, no. 1, pp. 126-128, 1998.

[4] V. Lecoeuche, M. W. Hathaway, D. J. Webb, C. N. Pannell, and D.A. Jackson, "20-km Distributed Temperature Sensor Based on Spontaneous Brillouin Scattering", IEEE Photonics Technology Letters, vol. 12, no. 10, pp. 1367-1369, 2000.

[5] P. C. Wait and A. H. Hartog, "Spontaneous Brillouin-Based Distributed Temperature Sensor Utilizing a Fiber Bragg Grating Notch Filter for the Separation of the Brillouin Signal", IEEE Photonics Technology Letters, vol. 13, no. 5, pp. 508-510, 2001.

[6] Y. T. Cho, M. Alahbabi, M. J. Gunning, and T. P. Newson, "50-km single-ended spontaneous-Brillouin -based distributed-temperature sensor exploiting pulsed Raman amplification", Optics Letters, vol. 28, no. 18, pp. 1651-1653, 2003.

[7] H. H. Kee, G. P. Lees, and T. P. Newson, "All-fiber system for simultaneous interrogation of distributed strain and temperature sensing by spontaneous Brillouin scattering," Optics Letters, vol. 25, no. 10 , pp. 695-697, 2000.

[8] M. N. Alahbabi, Y. T. Cho, and T. P. Newson, "100 $\mathrm{km}$ distributed temperature sensor based on coherent detection of spontaneous Brillouin backscatter", Measurement Science \& Technology, vol. 15, no. 8, pp. 1544-1547, 2004

[9] M. N. Alahbabi, Y. T. Cho, and T. P. Newson, "150-km-range distributed temperature sensor based on coherent detection of spontaneous Brillouin backscatter and in-line Raman amplification," Journal of the Optical Society of America B-Optical Physics, vol. 22, no. 6, pp. 1321-1324, 2005.

[10] Y. Koyamada, Y. Sakairi, N. Takeuchi, and S. Adachi, "Novel Technique to Improve Spatial Resolution in Brillouin Optcical Time-Domain Reflectometry," IEEE Photonics Technology letters, vol. 19, no. 23, pp. 1910-1912, 2007.

[11] M. A. Soto, G. Bolognini, and F. Di Pasquale, "30-km spontaneous-Brillouin distributed temperature sensor employing simplex-coding and low optical input power," in IEEE Conf. on Sensors 2008, Lecce, Oct. 26-29, pp. 282-285, 2008. 
[12] F. Wang, X. Zhang, Y. Lu, and Y. Dong, "Improvement of Spatial Resolution for Strain Measurement with Brillouin Optical Time-Domain Reflectometer by Fitting Method Based on Equivalent Optical Pulse," Acta Optica Sinica, vol. 28, no. 1, pp. 43-49, 2008(in Chinese).

[13] Xuping Zhang, Feng Wang, Yuangang Lu, and Rongrong Dou, "Improvement of spatial resolution of BOTDR by fitting Brillouin frequency shift distribution curve," presented at the 8th International Conference on Optical Communications and Networks (ICOCN 2009), Beijing, September 15-17, 2009(Invited Paper).

[14] Xuping Zhang, Feng Wang, and Yuangang Lu, "Fully Distributed Optical Fiber Sensor Based on Brillouin Effect," Laser \& Optoelectronics Progress, vol. 46, no. 11, pp. 60-64, 2009(in Chinese).

[15] Y. Lu, R. Dou, and X. Zhang, "Wideband Dectection of Spontaneous Brillouin Scattering Spectrum in Brillouin Optical Time-Domain Reflectometry," in Proc. SPIE, vol. 7158, pp. 715818, 2008.

[16] F. Wang, X. Zhang, Y. Lu, R. Dou, and X. Bao, "Spatial Resolution Analysis for DFT-Based Brillouin Optical Time Domain Reflectometry," Measurement Science \& Technology, vol. 20, no. 2, pp. 025202, 2009.

[17] Y. Lu, H. Liang, and X. Zhang, "Brillouin optical time-domain reflectometry based on Hadamard sequence probe pulse," presented at the 9th International Conference on Optical Communications and Networks (ICOCN 2010), Nanjing, October 24-26, 2010.

[18] L. Zou, X. Bao, Y. Wan, and L. Chen, "Coherent probe-pump-based Brillouin sensor for centimeter-crack detection," Optics Letters, vol. 30, no. 4, pp. 370-372, 2005. 